\title{
FILTRATIONS, ASYMPTOTIC AND PRÜFERIAN CLOSURES, CANCELLATION LAWS
}

\author{
HENRI DICHI AND DAOUDA SANGARE
}

(Communicated by Louis J. Ratliff, Jr.)

\begin{abstract}
Let $A$ be a commutative ring. For any filtration $f=\left(I_{n}\right)$ on the ring $A$ let $\bar{f}$ (resp. $P(f), f^{\prime}$ ) be the asymptotic (resp. prüferian, integral) closure of the filtration $f$. Then we have

$$
f \leq f^{\prime} \leq P(f) \leq \bar{f} .
$$

In this paper several examples to show that each relation in $(*)$ may be an equality or a strict inequality even in noetherian rings, are given. Some transfer properties (such as the property that a filtration be $A P$ or strongly $A P$ ) between the filtrations $f, P(f)$, and $\bar{f}$ are also given and negative answers are illustrated by some examples. This paper is closed by studying some cancellation laws concerning the prüferian closure of filtrations. In particular it is shown in the main theorem that if $f, g, h$ are filtrations on the noetherian ring $A$ such that $\sqrt{f} \subseteq \sqrt{h}$, if $P(f h) \leq P(g h)$ and if $h$ is strongly $A P$ then we have $P(f) \leq P(g)$. In this theorem the hypothesis " $h$ strongly $A P$ " cannot be weakened to " $h A P$ " as shown in Example 2.3(3).
\end{abstract}

\section{INTRODUCTION}

In [7] result 2.3.2 and Theorem 2.6 (Cancellation law), the authors misquoted what Rees has shown in pages 11-12 of his paper entitled: Asymptotic properties of ideals, preprint. Indeed they did not require the filtrations $f$ and $g$ to be noetherian. Our paper originated with the attempt to show that Theorem 2.6 (Cancellation law) of [7] holds for arbitrary filtrations $f$ and $g$ provided that the filtration $h$ be strongly $A P$. In addition we show here that this Cancellation law does not hold for arbitrary $h$ even if $h$ is $A P$. All rings are assumed to be commutative with an identity. $I, J, \ldots$ etc. will denote arbitrary ideals of the given ring $A$. For any filtration $f=\left(I_{n}\right)$ on the ring $A$, the asymptotic (resp. prüferian, integral) closure $\bar{f}$ (resp. $P(f), f^{\prime}$ ) of $f$ are defined in $\S 1.1$ (resp. 1.2) below. We have the following inequalities for a given filtration $f$

$$
f \leq f^{\prime} \leq P(f) \leq \bar{f} .
$$

In $\S 1$ of this paper we give some well-known relations between filtrations and

Received by the editors May 7, 1990 and, in revised form, July 20, 1990.

1980 Mathematics Subject Classification (1985 Revision). Primary 13A15, 13B20, 13 C99. 
pseudo-valuations that are needed later and we recall a cancellation law given by [8] concerning the asymptotic closure of filtrations.

In $\S 2$ we give various examples to show that the inequality between any two members in $(*)$ may be an equality or a strict inequality even in noetherian rings. We show also that the prüferian closure does not satisfy in general the same type of cancellation law as the asymptotic closure even if the filtration $h$ satisfies nice properties such as the $A P$ condition (see Example 2.3(3)).

We close this section with some transfer properties between $f, P(f)$, and $\bar{f}$. Although $P(f)$ and $\bar{f}$ are different in general, $P(f)$ is, however, very close to $\bar{f}$ (see 2.4(5)). We use this remark to show that $\bar{f}$ is $A P$ if and only if $P(f)$ is $A P$ (Proposition 2.5). However this equivalence is not true if we take "strongly $A P$ " in place of " $A P$ " as shown in Example 2.4(6).

In $\S 3$ we give a weak cancellation law concerning the prüferian closure of filtrations. Then we prove in the main theorem (see Theorem 3.3) that if $f, g, h$ are filtrations on the noetherian ring $A$ such that $\sqrt{f} \subseteq \sqrt{h}$, if $P(f h) \leq P(g h)$ and if $h$ is strongly $A P$, we have $P(f) \leq P(g)$.

In this theorem the hypothesis " $h$ strongly $A P$ " cannot be weakened to " $h A P$ " as shown in Example 2.3(3).

\section{ASYMPTOTIC CLOSURE OF FILTRATIONS}

1.1. Definitions. (1) A filtration on the ring $A$ is a sequence $f=\left(I_{n}\right)$ of ideals of $A$ such that $I_{0}=A, I_{n+1} \subseteq I_{n}$, and $I_{n} \cdot I_{m} \subseteq I_{n+m}$ for all $n, m \geq 0$. The set $\mathbb{F}(A)$ of all filtrations on the ring $A$ is ordered by $f=\left(I_{n}\right) \leq g=\left(J_{n}\right)$ if $I_{n} \subseteq J_{n}$ for any two elements $f, g$ in $\mathbb{F}(A)$. The product of the filtrations $f=\left(I_{n}\right)$ and $g=\left(J_{n}\right)$ is the filtration $f g=\left(I_{n} J_{n}\right)$.

(2) A pseudovaluation on the ring $A$ is a mapping $v: A \rightarrow \mathbb{R}_{+} \cup(\infty)$ such that

(i) $v(0)=\infty ; v(1)=0$

(ii) $v(x-y) \geq \min (v(x), v(y))$

(iii) $v(x y) \geq v(x)+v(y)$, for all $x, y \in A$;

here $\mathbb{R}_{+}$is the set of nonnegative real numbers.

(3) Let $v$ be a pseudovaluation on $A$. The homogeneous pseudovaluation associated with $v$ is the pseudovaluation $\bar{v}$ defined by $\bar{v}(x)=\lim _{n \rightarrow \infty} v\left(x^{n}\right) / n$ [4, Proposition 11.1]. We have $\bar{v} \geq v$.

(4) For any pseudovaluation $v$ on $A$, we put $[v](x)=[v(x)]$ for all $x \in A$, where $[v(x)]$ is the greatest integer less than or equal to the real number $v(x)$. $[v]$ is a pseudovaluation on $A$ that is integer-valued.

(5) We have $\overline{[v]}=\bar{v}$.

(6) With any filtration $f=\left(I_{n}\right) \in \mathbb{F}(A)$ we associate the pseudovaluation $v_{f}$ such that $v_{f}(x)=\sup \left\{n ; x \in I_{n}\right\}$ for all $x \in A$ (see [1] for generalizations of this concept).

For any ideal $I$ of $A$ we put $v_{I}=v_{f_{I}}$, where $f_{I}=\left(I^{n}\right)$ is the $I$-adic filtration; $v_{I}$ is called the $I$-adic pseudovaluation. 
(7) Conversely for any pseudovaluation $v$ on $A$ we put $f_{v}=\left(I_{v, n}\right)_{n \geq 0}$, where $I_{v, n}=\{x \in A ; v(x) \geq n\} . f_{v}$ is a filtration on $A$ and we have:

(8) $v_{f_{v}}=[v] ; f_{v_{f}}=f$.

(9) The asymptotic closure of the filtration $f$ is the filtration $\bar{f}$ associated with the homogeneous pseudovaluation $\bar{v}_{f} ; \bar{f}=f_{\bar{v}_{f}}$. We have:

(10) $v_{\bar{f}}=v_{f_{w}}=\left[\bar{v}_{f}\right]$, where $w=\bar{v}_{f}$. This follows from (8). We have also:

(11) $\bar{v}_{\bar{f}}=\left[\overline{\bar{v}}_{f}\right]=\overline{\bar{v}}_{f}=\bar{v}_{f}$. These are consequences of (5) and (10).

The following theorem is a part of Theorem 2.10 of [8].

1.2. Theorem. The operation $f \rightarrow \bar{f}$ is a semiprime operation on the set $\mathbb{F}(A)$. That is, for all filtrations $f$ and $g$ on $A$ we have:

(i) $f \leq \bar{f}$;

(ii) $\overline{\bar{f}}=\bar{f}$;

(iii) if $f \leq g$, then $\bar{f} \leq \bar{g}$;

(iv) $\bar{f} \bar{g} \leq \overline{f g}$.

Furthermore we have the following cancellation law:

Let $f, g, h \in \mathbb{F}(A)$ such that $\sqrt{f} \subseteq \sqrt{h}$, if $\overline{f h} \leq \overline{g h}$ then $\bar{f} \leq \bar{g}$.

\section{Prüferian Closure of Filtrations}

2.1. Definitions. (1) An element $x \in A$ is said to be integral over the filtration $f=\left(I_{n}\right)$ if $x$ satisfies an equation $x^{m}+a_{1} x^{m-1}+\cdots+a_{j} x^{m-j}+\cdots+a_{m}=0$, where $a_{j} \in I_{j}$ for all $j$.

(2) Let $I$ be an ideal of $A$. An element $x \in A$ which is integral over the $I$-adic filtration $f_{I}$ is said to be integral over $I$. We put $I^{\prime}=\{x \in A ; x$ is integral over $I\} . I^{\prime}$ is an ideal of $A$ and is called the integral closure of the ideal $I$. This is a well-known result.

(3) Let $k$ be an integer $\geq 1$. For any filtration $f=\left(I_{n}\right), f^{(k)}$ denotes the filtration $\left(I_{n k}\right)_{n \geq 0}$.

(4) Let $f=\left(I_{n}\right) \in \mathbb{F}(A)$ and let $k \geq 1$ be an integer. We put $P_{k}(f)=\{x \in$ $A ; x$ is integral over $\left.f^{(k)}\right\} . P_{k}(f)$ is the set of elements $x \in A$ such that $x^{m}+a_{1} x^{m-1}+\cdots+a_{j} x^{m-j}+\cdots+a_{m}=0$, for some integer $m \geq 1$, where $a_{j} \in I_{j k}$ for all $j$.

(5) The filtration $P(f)=\left(P_{n}(f)\right)$ is called the prüferian closure of $f$ following [3], or the weak integral closure of $f$ following [7] and the filtration $f^{\prime}=\left(I_{n}^{\prime}\right)$ is called the integral closure of $f$.

(6) In particular $P_{1}(f)$ is the set of elements $x \in A$ which are integral over $f^{(1)}=f$ and $P_{1}\left(f_{I}\right)=I^{\prime}$ for any ideal $I$ of $A$.

Let $f=\left(I_{n}\right)$ and $g=\left(J_{n}\right)$ be two filtrations on $A$.

(7) $g$ is said to be integral over $f$ if $g \leq P(f)$, that is, $J_{n} \subseteq P_{n}(f)$ for all $n \geq 1$.

(8) Now we will give a list of well-known filtrations that behave like $I$-adic filtrations and we will order them according to a diagram. Let $f=\left(I_{n}\right) \in \mathbb{F}(A)$. 
(a) $f$ is said to be $I$-good, where $I$ is an ideal of $A$, if $I \cdot I_{n} \subseteq I_{n+1}$ for all $n$ and $I \cdot I_{n}=I_{n+1}$ for all large $n$.

(b) $f$ is approximatable by powers and is called $A P$ for short in case there exists a sequence of positive integers $\left(k_{n}\right)$ such that $I_{k_{n} m} \subseteq I_{n}^{m}$ for all $n, m$ and $k_{n} / n \rightarrow 1$ as $n \rightarrow \infty$.

(c) $f$ is called strongly $A P$ if there exists an integer $k \geq 1$ such that $I_{n k}=I_{k}^{n}$ for all $n$, that is $f^{(k)}=f_{I_{k}}$.

(d) $f$ is said to be noetherian if the Rees ring $\mathscr{R}(A, f)=A\left[u, I_{1} X, I_{2} X^{2}\right.$, ...], where $X$ is an indeterminate and $u=X^{-1}$ is noetherian. This terminology is that introduced by D. Rees [10]. It has a different meaning in [2].

(e) $f$ is strongly noetherian if $A$ is noetherian and if there exists an integer $k \geq 1$ such that $I_{m} \cdot I_{n}=I_{m+n}$ for all $m, n \geq k$ (see [6]). We have in any ring $A$ :

$$
f I \text {-adic } \Rightarrow f I \text {-good } \Rightarrow f \text { strongly } A P \Rightarrow f A P \text {. }
$$

If $A$ is noetherian then $f=\left(I_{n}\right)$ is noetherian if and only if there exists an integer $k \geq 1$ such that $I_{n+k}=I_{n} I_{k}$ for all $n \geq k$. Furthermore each noetherian filtration on a noetherian ring is strongly $A P$, (see $[9,2])$. So in a noetherian ring $A$ we have the following diagram for any filtration $f$ :

$$
\begin{aligned}
& f I \text {-adic } \Rightarrow f I \text {-good } \Rightarrow f \text { strongly } A P \Rightarrow f A P \\
& \Downarrow \quad \Uparrow \\
& f \text { strongly } \Rightarrow f \text { noetherian } \\
& \text { noetherian }
\end{aligned}
$$

2.2. Relations between $f, f^{\prime}, P(f)$, and $\bar{f}$. We have

(1) $f \leq f^{\prime} \leq P(f) \leq \bar{f}$;

(2) $P\left(f^{\prime}\right)=(P(f))^{\prime}=P(f)$;

(3) $\bar{f}=\overline{f^{\prime}}=\overline{P(f)}$.

Proof. (1) is easy to show.

(2) We have $P(f) \leq P\left(f^{\prime}\right) \leq P(P(f))=P(f)$ and $P(f) \leq P(f)^{\prime} \leq$ $P(P(f))=P(f)$.

(3) follows from (1) and Theorem 1.2.

Each inequality in (1) may be an equality or a strict inequality. We have:

(4) For any ideal $I,\left(f_{I}\right)^{\prime}=f_{I}$ if and only if $I$ is normal (see [4, Chapter $11 ; 11])$.

(5) $\left(f_{I}\right)^{\prime}=P\left(f_{I}\right)$, as easily shown. Let $\bar{v}_{I}$ be the $I$-adic homogeneous pseudovaluation on the noetherian ring $A$ (see 1.1(3) and 1.1(6)) and let $k$ be an integer $\geq 1$. Then for each $x \in A$, we have:

(6) $\bar{v}_{I}(x) \geq k \Leftrightarrow x \in\left(I^{k}\right)^{\prime}$. In other words, $P\left(f_{I}\right)=\bar{f}_{I}$. For a generalization of (6) see [1, Theorem 5.6]. Another generalization of (6) has been given by [3, Proposition 4.7] as follows.

(7) If $A$ is noetherian and if the filtration $f$ is strongly $A P$ then $P(f)=\bar{f}$. 
2.3. Examples. Let $A=k[X]$, where $k$ is a field and $X$ an indeterminate

(1) Let $p$ be an integer $\geq 2$ and let $f=\left(I_{n}\right)$ where $I_{\dot{0}}=A$,

$$
I_{n}= \begin{cases}\left(X^{n}\right) & \text { if } n \geq p+1 \\ \left(X^{p}\right) & \text { if } 1 \leq n \leq p .\end{cases}
$$

It is easy to show that $f$ is a filtration which is strongly noetherian hence strongly $A P ; Y=X$ is integral over $f$ since $Y^{p}-X^{p}=0$ with $X^{p} \in I_{p}$. But $Y=X$ is not integral over $I_{1}=\left(X^{p}\right)$. Hence $I_{1}^{\prime} \neq P_{1}(f)$ and $f^{\prime} \neq P(f)$.

(2) Let $f=\left(I_{n}\right)$ where $I_{0}=A$ and $I_{n}=\left(X^{n+1}\right)$ for $n \geq 1 . f$ is a filtration on $A$ which is $A P$ (take $k_{n}=n+1$ for all $n$ ). We have $\bar{v}_{f}(X)=1$, hence $X \in I_{\bar{v}_{f, 1}}$. But it is easy to show that $X \notin P_{1}(f)$, therefore $P(f) \neq \overline{\bar{f}}$.

(3) Let $I=(X) ; I_{n}=\left\{\begin{array}{ll}I^{n} & \text { if } n \text { is even, } \\ I^{n+1} & \text { if } n \text { is odd, }\end{array} J_{0}=A ; J_{n}=I^{n+1}\right.$ if $n \geq 1$, and let $f=\left(I_{n}\right), g=h=\left(J_{n}\right) . f, g, h$ are filtrations on $A, f$ is noetherian, $g=h$ is $A P$. We have $\sqrt{f}=\sqrt{g}=\sqrt{h}=I$. If we put $f h=\left(K_{n}\right)$; $g h=\left(L_{n}\right)$, then $K_{2 n}=I^{4 n+1} ; K_{2 n-1}=I^{4 n} ; L_{n}=I^{2 n+2}$ for all $n \geq 1$.

To show that $P(f h) \leq P(g h)$ it is enough to have $f h \leq P(g h)$, i.e., $K_{n} \subseteq$ $P_{n}(g h)$ for all $n$. We have $K_{2 n-1}=I^{4 n}=L_{2 n-1} \subseteq P_{2 n-1}(g h) ; K_{2 n}=I^{4 n+1}$, $L_{4 n}=I^{8 n+2}$. If we take $Y=X^{4 n+1}$ then $Y^{2}-X^{8 n+2}=0$, with $X^{8 n+2} \in L_{4 n}$, therefore $Y \in P_{2 n}(g h)$, hence $K_{2 n} \subseteq P_{2 n}(g h)$ and $P(f h) \leq P(g h)$. But it is not true that $P(f) \leq P(g)$. For example $Y=X$ satisfies $Y^{2}-X^{2}=0$, with $X^{2} \in I_{2}$, that means $X \in P_{1}(f)$. But it is easy to show that $X \notin P_{1}(g)$, therefore $P_{1}(f)$ is not included in $P_{1}(g)$. This last example shows that the Cancellation law given in our Theorem 3.3 does not hold for arbitrary filtrations $f, g, h$ even if $h$ is $A P$.

2.4. Transfer properties. For any filtration $f$ on the noetherian ring $A$ the following implications are easily shown.

(1) $P(f)$ strongly $A P \Rightarrow f^{\prime}$ strongly $A P \Rightarrow f$ strongly $A P$. Furthermore if one of the above conditions is fulfilled, then we have:

(2) $\bar{f}=P(f)$ [3, Proposition 4.7]. Following [10, Introduction], for any filtration $f$ on the ring $A$ there exists a pseudovaluation $\left(v_{f}\right)^{*}$ on $A$ which satisfies:

(3) For $x \in A$ and $n \in \mathbb{N},\left(v_{f}\right)^{*}(x) \geq n \Leftrightarrow x \in P_{n}(f)$. Therefore $I_{\left(v_{f}\right)^{*}, n}=$ $P_{n}(f)$ for all $n$, hence $f_{\left(v_{f}\right)^{*}}=P(f)$ and $\left(v_{f}\right)^{*}=v_{f_{\left(v_{f}\right)^{*}}}=v_{P(f)}$. Following the same reference as above we have for $x \in A$ :

(4) $\left(v_{f}\right)^{*}(x) \leq \bar{v}_{f}(x)<\left(v_{f}\right)^{*}(x)+1$. An immediate consequence of (4) is

(5) $P_{n+1}(f) \subseteq I_{\bar{v}_{f, n+1}} \subseteq P_{n}(f)$, for all $n$. Relation (5) means that $P(f)$ is very close to $\bar{f}$. If in addition the filtration $f$ is noetherian then, as suggested by the referee, we have $\left[\bar{v}_{f}(x)\right]=\left(v_{f}\right)^{*}(x)$ (see e.g. $[10]$ ), hence $\bar{f}=P(f)$.

Let us remark that with the general hypothesis $\bar{f}=P(f)$ and $\bar{g}=P(g)$, Theorem 2.6 of [7] is a consequence of Theorem 2.10 of [8] which is our The- 
orem 1.2. Indeed if $P(f h)=P(g h)$ then $\overline{f h}=\overline{P(f h)}=\overline{P(g h)}=\overline{g h}$. If in addition $\sqrt{f}+\sqrt{g} \subseteq \sqrt{h}$ then by Theorem $1.2 P(f)=\bar{f}=\bar{g}=P(g)$.

We may ask whether the diagram (1) can be completed by the implication $\bar{f}$ strongly $A P \Rightarrow P(f)$ strongly $A P$. This would imply that $\bar{f}$ strongly $A P \Rightarrow f$ strongly $A P$. This last implication is not true as shown by the following

(6) Example. Let $A$ be a principal ideal domain and let $p$ be an irreducible element of $A$. Consider the filtration $f=\left(I_{n}\right)$, where $I_{0}=A, I_{n}=P^{n+1}$ for $n \geq 1$, with $P=p A$. We have $\bar{v}_{f}(p)=1$, therefore, $\bar{v}_{f}\left(p^{n}\right)=n$ for all $n$. Since $I_{\bar{v}_{f, n}} \subseteq P \subseteq I_{\bar{v}_{f, 1}}$, we have $P^{n}=I_{\bar{v}_{f, n}}$ for all $n$. It follows that $\bar{f}=f_{P}$ is $P$-adic hence strongly $A P$. But $f$ is not strongly $A P$.

However, we have

2.5. Proposition. Let $f \in \mathbb{F}(A)$. Then $\bar{f}$ is $A P$ if and only if $P(f)$ is $A P$. Proof. Suppose that $\bar{f}=\left(J_{n}\right)$ is $A P$. Then there exists a sequence $\left(k_{n}\right)$ of integers such that $\lim _{n \rightarrow \infty} k_{n} / n=1$ and $J_{k_{n} m} \subseteq J_{n}^{m}$ for all $n, m$. By (5) we have $P_{k_{n} m}(f) \subseteq J_{k_{n} m} \subseteq J_{n}^{m} \subseteq\left(P_{n-1}(f)\right)^{m}$. Therefore $P_{k_{n+1} m}(f) \subseteq\left(P_{n}(f)\right)^{m}$, with $\lim _{n \rightarrow \infty} k_{n+1} / n=1$ and $P(f)$ is $A P$. Conversely suppose that $P(f)$ is $A P$. Let $\left(k_{n}\right)$ be a sequence of integers such that $P_{k_{n} m}(f) \subseteq\left(P_{n}(f)\right)^{m}$ for all $n, m$ and $\lim _{n \rightarrow \infty} k_{n} / n=1$. We have for all $m \geq 1, J_{\left(k_{n}+1\right) m} \subseteq J_{k_{n} m+1} \subseteq$ $P_{k_{n} m} \subseteq\left(P_{n}(f)\right)^{m} \subseteq J_{n}^{m}$ by (5). So, $\bar{f}$ is $A P$ since $\lim _{n \rightarrow \infty}\left(k_{n}+1\right) / n=1$.

2.6. To close this section, we know [3, Proposition 4.7] that for any strongly $A P$ filtration $f$ on the noetherian ring $A$, we have $P(f)=\bar{f}$. But the converse implication is not true, that is there exist filtrations $f$ that are not strongly $A P$ (even not $A P$ ) such that $P(f)=\bar{f}$ as shown in the following example. This fact is not surprising because for any filtration $f, P(f)$ is very close to $\bar{f}$.

Example. Let $I$ be a maximal ideal of the ring $A$ such that $I^{2} \neq I$ and let $f=\left(I_{n}\right)$ where $I_{0}=A, I_{n}=I$ for all $n \geq 1 . f$ is a filtration which satisfies $f=f^{\prime}=P(f)=\bar{f}$. Indeed, by $2.2(1)$ we need only to show that $\bar{f} \leq f$, which is easily done. However $f$ is not $A P$.

\section{CANCELlation LAWS FOR THE PRÜFERIAN CLOSURE OF FILTRATIONS}

In this section we will give first a weak cancellation law for the prüferian closure (Corollary 3.2) and secondly a cancellation law which is like that verified by the asymptotic closure (Theorem 3.3). The next proposition is an adaptation of [5, Theorem 3].

3.1. Proposition. Let $f=\left(I_{n}\right), g=\left(J_{n}\right)$, and $h=\left(K_{n}\right)$ be filtrations on $A$. Suppose that $K_{n}$ is finitely generated for all $n$. If $\sqrt{f} \subseteq \sqrt{h}$ and if $f h \leq g h$, then $f \leq g^{\prime}$.

Proof. We need only to show that $I_{n} \subseteq J_{n}^{\prime}$ for all $n$, where $J_{n}^{\prime}$ is the integral closure of the ideal $J_{n}$. Suppose that $K_{n}$ is generated by $c_{1}, c_{2}, \ldots, c_{r}$. Let 
$x \in I_{n}$. Then $c_{i} x=\sum_{j=1}^{r} b_{i j} c_{j}$, where $b_{i j} \in J_{n}$ for $i=1,2, \ldots, r$. Hence $\sum_{j=1}^{r}\left(\delta_{i j} x-b_{i j}\right) c_{j}=0$, where $\delta_{i i}=1$ and $\delta_{i j}=0$ for $j \neq i$. Consider the matrix $M=\left(\delta_{i j} x-b_{i j}\right)$. We have $M X=0$, where ${ }^{t} X=\left(c_{1}, \ldots, c_{r}\right)$. Let $d$ be the determinant of $M$. Then it is well known that there exists a matrix $\tilde{M}$ such that ${ }^{t} \tilde{M} M X=d X=0$, where ${ }^{t} \tilde{M}$ is the transpose of $\tilde{M}$. It follows that $d c_{i}=0$ for all $i$ and $d K_{n}=0$. We have $d x \in \sqrt{d I_{n}}=$ $\sqrt{d A} \cap \sqrt{f} \subseteq \sqrt{d A} \cap \sqrt{h}=\sqrt{d K_{n}}=\sqrt{0}$. So there exists an integer $s \geq 1$ such that $d^{s} x^{s}=0$. Finally $d=x^{r}+a_{1} x^{r-1}+\cdots+a_{j} x^{r-j}+\cdots+a_{r}$, where $a_{j} \in J_{n}^{j}$ for $j=1,2, \ldots, r ; d^{s}=x^{r s}+b_{1} x^{r s-1}+\cdots+b_{j} x^{r s-j}+\cdots+b_{r s}$, with $b_{j} \in J_{n}^{j}$ for $j=1,2, \ldots, r$. It follows that $0=d^{s} x^{s}=x^{r s+s}+b_{1} x^{r s}+b_{1} x^{r s+s-1}+\cdots+b_{r s} x^{s}$, hence $x \in J_{n}^{\prime}$.

3.2. Corollary. Let $f, g, h$ be filtrations on the noetherian ring $A$ such that $\sqrt{f}+\sqrt{g} \subseteq \sqrt{h}$. If $P(f) P(h)=P(g) P(h)$, then $P(f)=P(g)$.

Proof. We have $\sqrt{P(f)}=\sqrt{f} \subseteq \sqrt{h}$ and $(P(f))^{\prime} \leq(P(g))^{\prime}$ by Proposition 3.1. Hence by 2.2(2) $P(f) \leq P(g)$. The conclusion follows by symmetry.

3.3. Theorem. Let $f, g, h$ be filtrations on a noetherian ring $A$ such that $\sqrt{f} \subseteq \sqrt{h}$. If $h$ is strongly $A P$ and if $P(f h) \leq P(g h)$, then $P(f) \leq P(g)$.

Proof. Suppose first that $f$ and $g$ are strongly $A P$. Since the product of two strongly $A P$ filtrations is strongly $A P$ the conclusion follows by Theorem 1.2 and [3, Proposition 4.7].

Now suppose that $f=\left(I_{n}\right)$ and $g=\left(J_{n}\right)$ are arbitrary filtrations. For all integers $m \geq 1$, let $f_{m}$ be the smallest filtration whose first $m+1$ terms are $A, I_{1}, I_{2}, \ldots, I_{m} \cdot f_{m}$ is noetherian [2, Proposition 2.8]. Hence $f_{m}$ is strongly $A P$ [9, Remark 2.4.3]. We have $f h \leq P(g h)$ which implies $f_{m} h \leq P(g h)$ since $f_{m} \leq f$. Let $f_{m} h=\left(K_{n}\right) ; f_{m} h$ is strongly $A P$. There exists an integer $r \geq 1$ such that $\left(f_{m} h\right)^{(r)}=f_{K_{r}}$. Moreover $f_{m} h$ is integral over $g h=\left(L_{n}\right)$, hence $K_{r}$ is integral over $(g h)^{(r)}$. Suppose that $K_{r}$ is generated by $x_{1}, x_{2}, \ldots, x_{t}$, then there exists an integer $N \geq 1$ such that:

$$
\begin{aligned}
& x_{i}^{N}+a_{i, 1} x_{i}^{N-1}+\cdots+a_{i, j} x_{i}^{N-j}+\cdots+a_{i, N}=0, \text { where } a_{i j} \in L_{j r} \\
& \text { for all } j=1,2, \ldots, N \text { and } i=1,2, \ldots, t .
\end{aligned}
$$

Let $g_{N r}$ be the smallest filtration whose first $N r+1$ terms are $A, J_{1}, J_{2}, \ldots$, $J_{N r}$. As shown above $g_{N r}$ is noetherian, hence strongly $A P$. By $(*), K_{r}$ is integral over $\left(g_{N r}\right)^{(r)}$, which implies that $f_{m} h$ is integral over $g_{N r} h$ by [3, Corollary 4.3]. Therefore $P\left(f_{m} h\right) \leq P\left(g_{N r} h\right)$. As $\sqrt{f_{m}}=\sqrt{f} \subseteq \sqrt{h}$, then it follows from the first part of this proof that $f_{m} \leq P\left(f_{m}\right) \leq P\left(g_{N r}\right) \leq P(g)$ for all $m \geq 1$. Hence $I_{m} \subseteq P_{m}(g)$ for all $m$ and $P(f) \leq P(g)$.

3.4. Corollary. Let $f, g, h$ be filtrations on the noetherian ring $A$ such that $\sqrt{f}+\sqrt{g} \subseteq \sqrt{h}$. If $h$ is strongly $A P$ and if $P(f h)=P(g h)$, then $P(f)=P(g)$. 
Proof. This corollary is an immediate consequence of Theorem 3.3.

3.5. Remark. As shown in Example 2.3(3), the Cancellation law 3.3 does not hold with the weaker condition " $h A P$. ."

\section{ACKNOWLEDGMENT}

The authors would like to express their gratitude to the referee for his criticism on the first version of this note.

\section{REFERENCES}

1. P. Ayegnon and D. Sangare, Generalized Samuel numbers and AP filtrations, J. Pure Appl. Algebra 65 (1990), 1-13.

2. W. Bishop, A theory of multiplicity for multiplicative filtrations, J. Reine Angew. Math. 277 (1975), 8-26.

3. H. Dichi, Integral dependence over a filtration, J. Pure Appl. Algebra 58 (1989), 7-18.

4. S. McAdam, Asymptotic prime divisors, Lecture Notes in Math., vol. 1023, Springer-Verlag, Berlin, 1983.

5. M. Nagata, Note on a paper of Samuel concerning asymptotic properties of ideals, Mem. Coll. Sci. Kyoto Univ. Ser. A, vol. 30, Mathematics, No. 2, 1957, pp. 165-175.

6. J. S. Okon, Prime divisors, analytic spread and filtrations, Pacific J. Math. 113 (1984), 451-462.

7. J. S. Okon and L. J. Ratliff, Jr., Filtrations, closure operations and prime divisors, Math. Proc. Cambridge Philos. Soc. 104 (1988), 31-46.

8. J. W. Petro, Some results in the theory of pseudo valuations, Ph.D., State Univ. of Iowa, Graduate College, 1961.

9. L. J. Ratliff, Jr., Notes on essentially powers filtrations, Michigan Math. J. 26 (1979), 313324.

10. D. Rees, Semi noether filtrations: I, J. London Math. Soc. (2) 37 (1988), 43-62.

11. Shiro Goto, Integral closedness of complete intersection ideals, J. Algebra 108 (1987), 151160.

Département de MathematiQues, Université d’Abidjan, FAST, 22 BP 582 Abidjan 22 , CôTE D'IVOIRE 